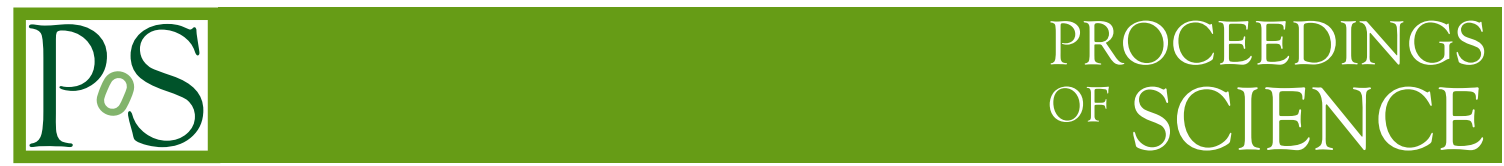

\title{
Latest single top differential cross section measurements at CMS
}

\section{Víctor Rodríguez Bouza (on behalf of the CMS Collaboration) ${ }^{a, *}$}

${ }^{a}$ Universidad de Oviedo, Instituto Universitario de Ciencias y Tecnologías Espaciales de Asturias (ICTEA), Asturias (Spain)

E-mail: victor.rodriguez.bouza@cern.ch

Single top quark production is the subleading production process of top quarks at the LHC after the top quark pair production. The latest differential measurements of single top quark production cross sections are presented using data collected by the CMS detector at a center-of-mass energy of $13 \mathrm{TeV}$. The cross sections are measured as a function of various kinematic observables of the top quarks and the jets and leptons of the events in the final state. The results are confronted with precise theory calculations.

40th International Conference on High Energy physics - ICHEP2020

July 28 - August 6, 2020

Prague, Czech Republic (virtual meeting)

\footnotetext{
${ }^{*}$ Speaker
} 


\section{Introduction and methodology}

The top quark is a relevant fermion inside the standard model (SM) due to its high mass, thanks to which it plays an important role in stabilising the Higgs field. The set of processes where the top quark is singly produced is called single top. They display at least one Wtb vertex, which allows to probe the $\mathrm{V}_{\mathrm{tb}}$ element of the CKM matrix. In the case where the $\mathrm{W}$ boson is produced on-shell in the final state, the process is called tW. When considered at next-to-leading-order, some of its final states also appear in the top quark pair production process, leading to double-counting issues when simulating both process separately. Two methods are used in the field to avoid this, modifying the simulation of the $\mathrm{tW}$ process: the diagram removal (DR), where Feynman diagrams with two on-shell tops are removed from the calculation, and the diagram subtraction (DS), where an artificial term is added to the cross-section calculation to locally erase those contributions. Recently, differential cross sections measurements of this channel at $\sqrt{s}=13 \mathrm{TeV}$ have been made public [1] by the CMS Collaboration, and it is the analysis described in this document.

Our event selection maximises signal presence by choosing leptonic events with one electron and one muon, of opposite charge. The most energetic lepton in the transverse plane must have $\mathrm{p}_{\mathrm{T}}>25 \mathrm{GeV}$ and the other $\mathrm{p}_{\mathrm{T}}>20 \mathrm{GeV}$. The trigger selection is a combination of di-lepton and single-lepton ones. The classification of the events depending on the number of jets and $b$ jets in each event allows us to reduce the background contribution, which in the best case is roughly five times that of the signal (see left plot from Fig. 1). We focus on events that present a jet that must also be tagged as coming from a bottom quark $(1 \mathrm{j} 1 \mathrm{~b})$. We also veto the presence of loose jets (right plot of Fig. 1), that is, those with $20 \mathrm{GeV}<\mathrm{p}_{\mathrm{T}}<30 \mathrm{GeV}$.

We extract the signal by subtracting the predicted background from the data and afterwards we unfold six distributions to a particle level fiducial region very closely defined to the one used for signal selection at detector level. The chosen variables are the $\mathrm{p}_{\mathrm{T}}$ of the leading lepton and the jet, the difference in the $\varphi$ angle between the two leptons, the momentum in the $z$ axis of the electron, muon and jet system, the invariant mass of the two leptons and jet system and the transverse invariant mass of the two leptons, jet and $\mathrm{p}_{\mathrm{T}}^{\mathrm{miss}}$ system. No regularisation nor area constraint was deemed necessary for the unfolding, done using TUnfold [2]. The result is normalised to the fiducial cross section, and systematic uncertainties are propagated by repeating the process for each one.

\section{Result and discussion}

The final result for all observables is compared with generators as can be seen for the case of the leading lepton $\mathrm{p}_{\mathrm{T}}$ in Fig. 2 and the agreement between the expectations and the data is fairly good for all of them. Both DR and DS methods used for the tW process show compatible results. The uncertainties, (from $15-40 \%$ in the bulks up to $25-100 \%$ in the tails), are essentially of systematic origin and mainly come from the large background, with main sources being jet-related (e.g. jet energy scale) or from modelling (e.g. matrix element/parton shower matching).

\section{References}

[1] CMS Collaboration, "Measurement of differential cross sections for single top quark production in association with a W boson at $\sqrt{s}=13 \mathrm{TeV}$ ", CMS Physics Analysis Summary 

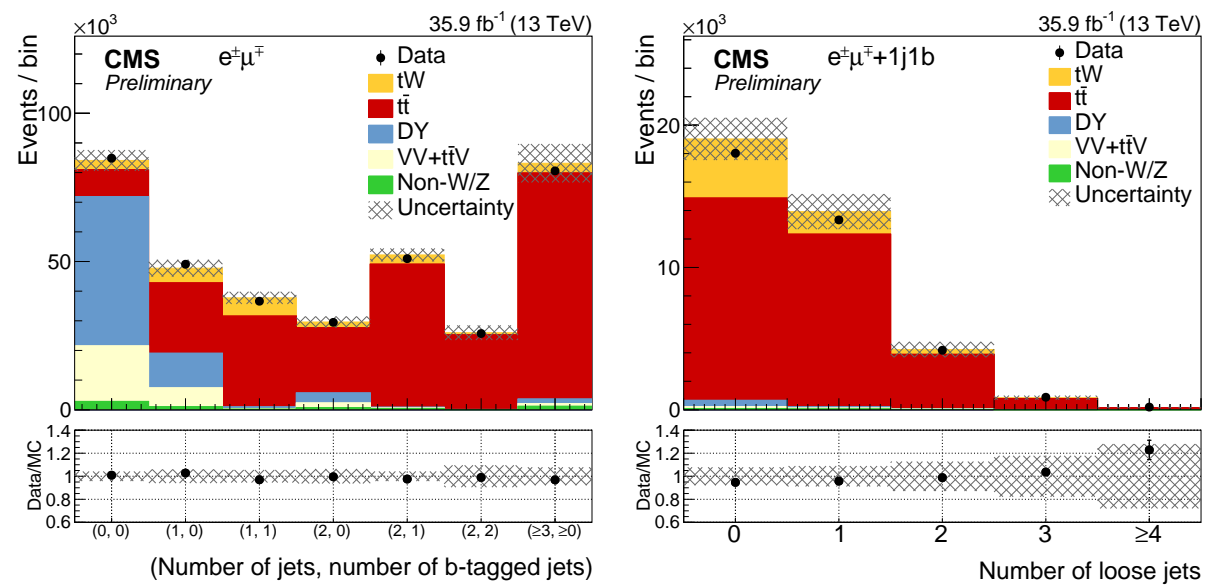

Figure 1: Yields observed in data, compared with expectations from simulation, depending on the number of jets and number of b-tagged jets for events passing the baseline dilepton selection (left) and on the number of loose jets from the $(1,1)$ bin of the previous distribution (right) The error band includes in the right plot all uncertainty sources whereas in the left plot the background normalisation ones are left aside. The bottom of each panel shows the ratios of data to the sum of the expected yields. [1]
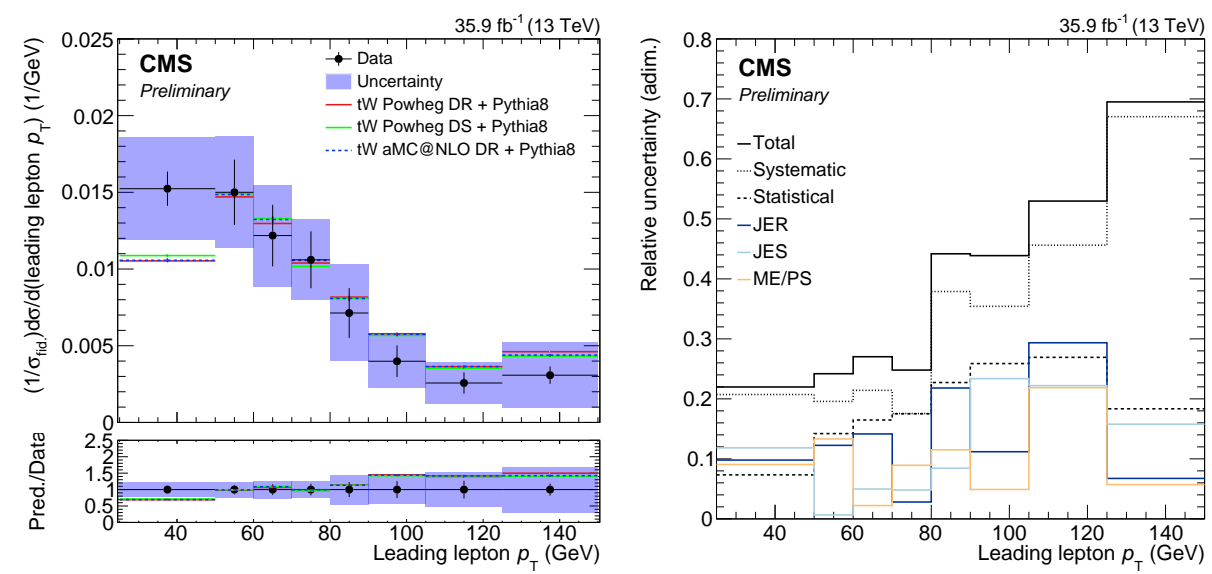

Figure 2: Left: normalised differential $\mathrm{tW}$ production cross section as a function of the $\mathrm{p}_{\mathrm{T}}$ of the leading lepton. The solid band represents the total uncertainty. Predictions from POWHEG and MadGraph5_aMC@NLO are shown. In the bottom panel, the ratio between predictions and data is shown. Right: total, systematic, statistical and individual leading (with its strength evaluated averaging each over all bins) uncertainties [1].

CMS-PAS-TOP-19-003, CERN, 2020, https://cds.cern.ch/record/2712818.

[2] S. Schmitt, "TUnfold, an algorithm for correcting migration effects in high energy physics", Journal of Instrumentation 7 (2012) 10.

\section{Acknowledgments}

Partially publicy funded by Ministerio de Ciencia e Innovación (Gobierno de España) through the “Ayudas para contratos predoctorales para la formación de doctores 2019" program. 